\title{
GROUP PRESENTATIONS CORRESPONDING TO SPINES OF 3-MANIFOLDS. III
}

BY

\author{
R. P. OSBORNE AND R. S. STEVENS
}

\begin{abstract}
Continuing after the previous papers of this series, attention is devoted to RR-systems having two towns (i.e., to compact 3-manifolds with spines corresponding to group presentations having two generators). An interesting kind of symmetry is noted and then used to derive some useful results. Specifically, the following theorems are proved:

THEOREM 1. Let $\phi$ be a group presentation corresponding to a spine of a compact orientable 3-manifold, and let $w$ be a relator of $\phi$ involving just two generators $a$ and $b$. If $w$ is cyclically reduced, then either

(a) $w$ can be "written backwards" (i.e., if $w=a^{m_{1}} b^{m_{1}} a^{m_{2}} b^{n_{2}} \ldots a^{m_{*}} b^{n_{*}}$, then $w$ is a cyclic conjugate of $\left.b^{n_{k}} a^{m_{k}} \ldots b^{n_{2}} a^{m_{2}} b^{n_{1}} a^{m_{1}}\right)$, or

(b) $w$ lies in the commutator subgroup of the free group on $a$ and $b$.

THEOREM 2. (Loose translation). If $\phi$ is a group presentation with two generators and if the corresponding 2-complex $K_{\phi}$ is a spine of a closed orientable 3-manifold then, $K_{\phi}$ is a spine of a closed orientable 3-manifold if and (except for two minor cases) only if $\phi$ has two relators and among the six allowable types of syllables (3 in each generator), exactly four occur an odd number of times. Further, each of the two relators can be "written backwards."
\end{abstract}

In [3] RR-systems were introduced and were shown to contain much useful information about the relationship between group presentations and spines of orientable 3-manifolds. In this paper we devote our attention to RR-systems having two towns and correspondingly to group presentations with two generators. We observe that many of these RR-systems have a certain kind of symmetry. By means of this symmetry we will derive some useful results. In particular, we will establish the following:

THEOREM 1. Let $\varphi$ be a group presentation corresponding to a spine of a compact orientable 3-manifold, and let $w$ be a relator of $\varphi$ involving just two generators $a$ and $b$. If $w$ is cyclically reduced, then $w$ can be "written backwards" or lies in the commutator subgroup of the free group on $a$ and $b$. (Specifically, $w$ can be "written backwards" if $w=a^{m_{1}} b^{n_{1}} a^{m_{2}} b^{n_{2}} \ldots a^{m_{k}} b^{n_{k}}$ is a cyclic conjugate of $\hat{w}=b^{n_{k}} a^{m_{k}} \ldots b^{n_{2}} a^{m_{2}} b^{n_{1}} a^{m_{1}}$.) (Cf. [5].)

Received by the editors April 20, 1976.

AMS (MOS) subject classifications (1970). Primary 57A10; Secondary 55A25.

Key words and phrases. Compact orientable 3-manifold, spine, 2-complex, group presentation, $P$-graph. 
We will refer to a station in a town of a RR-system as being an odd (even) station if it has an odd (even) number of tracks.

THEOREM 2 (MODIFIED). Let $R$ be a two-town $R R$-system in which every route meets both towns. Then every 3-manifold obtained from $R$ has a 2-sphere boundary if and only if $R$ has two companies and four odd stations.

Loosely translated, Theroem 2 says that if $\varphi$ is a two-generator presentation whose 2-complex $K_{\varphi}$ is a spine of a compact orientable 3-manifold, then $K_{\varphi}$ is a spine of a closed orinetable 3-manifold if and only if $\varphi$ has two relators and among the six allowable types of syllables ( 3 in each generator), exactly four occur an odd number of times. Moreover, each of the two relators can be "written backwards."

1. Development of the results. Inasmuch as we draw very heavily on the results and techniques of [3], we assume that the reader is familiar with them as well as the notation used there. Our primary results apply only for compact orientable 3-manifolds with spines having just two 1-cells. However, we will make use of two lemmas which apply to such manifolds with spines having any number of 1-cells. Accordingly, we give these lemmas first.

LEMMA 1. Let $\varphi=\left\langle a_{1}, \ldots, a_{n} \mid w_{1} \ldots, w_{n}\right\rangle$ be a group presentation that corresponds to a spine of a compact orientable 3-manifold $M$. For each $i=$ $1, \ldots, n$, let $\bar{w}_{i}$ denote $w_{i}$ abelianized. If the $w_{i}$ 's can be reordered so that $\bar{w}_{1} \neq 1$ and each $\bar{w}_{i}, i=2, \ldots, n$, is not a consequence of $\bar{w}_{1}, \ldots, \bar{w}_{i-1}$ in the free abelian group on $a_{1}, \ldots, a_{n}$, then $\varphi$ corresponds to a spine of a closed orientable 3-manifold.

Proof. Let the reordering of the $\bar{w}_{i}$ 's be done. Construct a sequence $M_{0}, M_{1}, \ldots, M_{n}$ of compact orientable 3-manifolds as follows: Let $M_{0}$ be a handlebody of genus $n$, and let $\gamma_{1}, \ldots, \gamma_{n}$ be disjoint simple closed curves in $\partial M_{0}$, each $\gamma_{i}$ corresponding to the relator $w_{i}$. Construct $M_{i}$ from $M_{i-1}$ by attaching a thickened disc (or 2-handle) along $\gamma_{i}$ (see [3] and [4]). We show that $\partial M_{n}=S^{2}$ by arguing inductively that each $\partial M_{i}$ is connected (and thus is a closed orientable 2-manifold of genus $n-1$ ).

Consider $\gamma_{i} \subset \partial M_{i-1}$. Since $\bar{w}_{i}$ is not a consequence of $\bar{w}_{1}, \ldots, \bar{w}_{i-1}$ (or in the case $i=1$ : since $\bar{w}_{1} \neq 1$ ), it follows that $\gamma_{i}$ is not homologically trivial in $\partial M_{i-1}$. Hence $\gamma_{i}$ does not separate $\partial M_{i-1}$. It follows that $\partial M_{i}$ is connected. Since an annulus has been removed from $\partial M_{i-1}$ and replaced by two discs to obtain $\partial M_{i}$, it follows that the genus of $\partial M_{i}$ is 1 less than that of $\partial M_{i-1}$. This completes the induction, and the lemma is proved.

Definition 1 . Let $R$ be any RR-system. Denote by $\mathfrak{N}_{R}$ the set of all compact orientable 3-manifolds with nonempty boundary that originate from $R$. It was shown in [3, Theorem 7.7] that every $M \in \mathfrak{N}_{R}$ has a 2-sphere 
boundary (so that we may "close" $M$ by attaching a 3-cell), or no $M \in \mathfrak{T}_{R}$ has a 2-sphere boundary. In the event that every $M \in \Re_{R}$ has a 2-sphere boundary, we will say that $\Re_{R}$ consists of closed manifolds. It was also shown in [3] that $\Re_{R}$ consists of closed manifolds if and only if (1) $R$ has equally many towns and companies and (2) $R$ is $R$-connected.

LEMMA 2. Let $R$ be a nonempty $R R$-system in which each town has all three of its stations of the same parity. Then $\mathfrak{N}_{R}$ does not consist of closed manifolds.

Proof. We may assume that $R$ has equally many towns and companies. Thus, since $R$ is nonempty, there is at least one town and one company. We color the components of $S^{2}-R$ (i.e., the open 2-cells remaining after all the towns and routes are removed) black and white so that no two components bordering on a common route have the same color. This is possible since every town has an even number of routes meeting it. We show that no white component is $R$-contiguous with any black component. It will then follow that $R$ is not $R$-connected; i.e., that $\mathfrak{T}_{R}$ does not consist of closed manifolds.

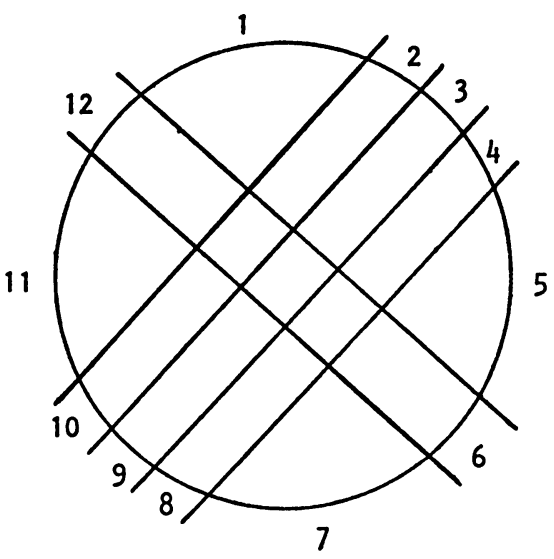

A town with all even stations (an empty station is considered to be even).

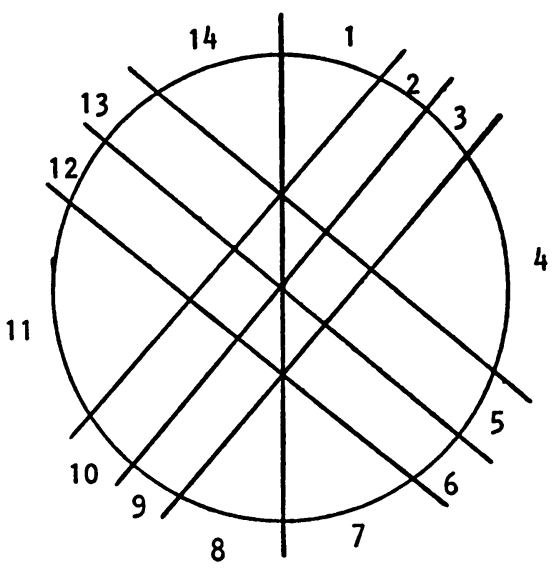

A town with all odd stations.

FIGURE 1b

\section{FIGURE 1a}

The odd and even numbers respectively indicate black and white components.

\section{FIGURE 1}

Since $R$-contiguity is defined in terms of a single town, it will suffice to argue this point over a single town. We say that a component of $S^{2}-R$ is of type A (of type B) with respect to the town if it borders on the town between two tracks of the same station (of different stations). A component of type A is $R$-contiguous (via the town) to a unique other component that is also of 
type A. Since all three stations have the same parity, it follows that these two components have the same color (e.g., see regions 3 and 9 in Figures la and $1 b)$. A component of type $\mathrm{B}$ is $R$-contiguous only to other components of type $B$. If the town has all even stations, then all type $B$ components must have the same color (see regions 1, 5, 7,11 in Figure 1a). If the town has all odd stations, then there are six components of type B, of which three are black and three are white (see 1, 7, 11, and 4, 8, 14, respectively, in Figure lb). It is easy to see that two of these type $\mathrm{B}$ components are $R$-contiguous (via the town) if and only if they have the same color. This completes the proof.

Henceforth we consider $R$ to be a two-town RR-system.

Definition 2. Let $R$ be a two-town RR-system in which every route meets both towns. We introduce a spherical coordinate system on $S^{2}$ so that (1) the boundaries of the towns are "latitudes" with the "north pole" in the center of one town and the "south pole" in the center of the other, and (2) the routes lie along equally spaced "meridians." Then a $180^{\circ}$ rotation of $S^{2}$ about the "polar axis" defines a nontrivial mapping of $R$ onto itself. This mapping will henceforth be referred to as the symmetry of $R$. Note that the symmetry of $R$ induces a nontrivial permutation of the abstract syllables in an abstract presentation corresponding to $R$.

Let $\eta$ denote the symmetry of $R$. If $\mu$ is any track in either town of $R$, then $\mu$ and $\eta(\mu)$ are tracks in the same station. If $x$ and $y$ are the endpoints of $\mu$, and $\mu$ is oriented from $x$ to $y$ while $\eta(\mu)$ is oriented from $\eta(x)$ to $\eta(y)$, then $\mu$ and $\eta(\mu)$ have opposite orientations in the station.

Definition 3. Given a (possibly abstract) word $W$, we use $\hat{W}$ to denote $W$ written backwards; e.g., if $W=a^{m_{1}} b^{n_{1}} a^{m_{2}} b^{n_{2}} \ldots a^{m_{k}} b^{n_{k}}$, then $\hat{W}=$ $b^{n_{k}} a^{m_{k}} \ldots b^{n_{2}} a^{m_{2}} b^{n_{1}} a^{m_{1}}$. If $W$ is a cyclic conjugate of $\hat{W}$, then we say that $W$ can be written backwards.

Lemma 3. Suppose that $R$ is a reduced $R R$-system with two towns and one company involving both towns. Let $W$ be an abstract word corresponding to that company. Then either (1) $W$ is of the form $U \hat{U}^{-1}$ and all stations of $R$ are even, or (2) $W$ can be written backwards and $R$ has exactly two odd stations.

Proof. Let $\mu_{0}$ denote the track on which we started our "train ride" to obtain $W$, and orient $\mu_{0}$ according to the direction of the "train ride." Let $\tilde{W}$ be the abstract word obtained from $R$ by starting from $\eta\left(\mu_{0}\right)$ and traveling in the direction indicated by the orientation of $\eta\left(\mu_{0}\right)$. An easy induction argument shows that $\tilde{W}$ is obtained from $W$ by merely changing the sign on each syllable; i.e., $\tilde{W}=W^{-1}$. Since $W$ and $\tilde{W}$ are obtained from the same company, it follows that $\tilde{W}$ is a cyclic conjugate of either $W$ or $W^{-1}$. The lemma follows. 
THEOREM 1. Let $\varphi$ be a group presentation corresponding to a spine of $a$ compact orientable 3-manifold, and let $w$ be a relator of $\varphi$ involving just two generators $a$ and $b$. If $w$ is cyclically reduced, then $w$ can be written backwards or lies in the commutator subgroup of the free group on $a$ and $b$.

Proof. From [1] and [4] we know that the $P$-graph $P_{\varphi}$ has a faithful embedding in $S^{2}$. Moreover, if $\varphi^{\prime}=\langle a, b \mid w\rangle$, then $P_{\varphi^{\prime}}$ inherits from $P_{\varphi}$ a faithful embedding in $S^{2}$. Thus (by [3, Theorem 1.6]), there is a two-town RR-system from which $\varphi$ originates. Since $w$ is cyclically reduced and $R$ has just two towns, $R$ is equivalent (via a sequence of RR-cancellations) to a reduced RR-system $R^{\prime}$ from which $\varphi^{\prime}$ originates. We apply Lemma 3 to $R^{\prime}$ to observe that the abstract relator $W$ is of the form $W=U \tilde{U}^{-1}$ or can be written backwards. Since $w$ is obtained from $W$ by merely substituting specific values for the exponents on the syllables, it follows that $w$ is in the commutator subgroup or can be written backwards.

Notation. If $C$ is a company in some RR-system $R$, then we write $s(C)$ to denote the number of tracks of $C$.

Definition 4. Let $R$ be a RR-system. The RR-system $R^{\prime}$ will be called a sub-RR-system of $R$ if $R^{\prime}$ is obtained from $R$ by deleting some of its companies. If the companies of $R$ are $C_{1}, \ldots, C_{n}$, then $R_{i}$ denotes the sub-RR-system of $R$ whose only comapny is $C_{i}, i=1, \ldots, n$.

THEOREM 2. Let $R$ be a reduced $R R$-system with two towns. Then $\mathfrak{T}_{R}$ consists of closed manifolds if and only if $R$ has exactly two companies (denoted $C_{1}$ and $C_{2}$ so that $s\left(C_{1}\right) \leqslant s\left(C_{2}\right)$ ) and one of the following holds:

(1) $1=s\left(C_{1}\right)=s\left(C_{2}\right)$ and each town has one track.

(2) $1=s\left(C_{1}\right)=\left\langle s\left(C_{2}\right)\right.$ and the sub-RR-system $R_{2}$ has two odd stations.

(3) $1<s\left(C_{1}\right) \leqslant s\left(C_{2}\right)$ and $R$ has four odd stations.

Proof. First suppose that $\Re_{R}$ consists of closed manifolds. Then $R$ is $R$-connected and has equally many towns and companies. Hence $R$ has two companies $C_{1}$ and $C_{2}$ which we label so that $1 \leqslant s\left(C_{1}\right) \leqslant s\left(C_{2}\right)$. We consider three cases.

Case 1. $1=s\left(C_{1}\right)=s\left(C_{2}\right)$. In this case we see that each town must have one track, for if both tracks are in the same town, then they must be in the same station. This implies that all stations are even, contradicting Lemma 2.

Case 2. $1=s\left(C_{1}\right)<s\left(C_{2}\right)$. Since $R$ is $R$-connected, it follows that $R_{2}$ is $R$-connected. By Lemma 3 it follows that the abstract relator $W_{2}$ obtained from $C_{2}$ can be written backwards and that $R_{2}$ has two odd stations.

Case 3. $1<s\left(C_{1}\right) \leqslant s\left(C_{2}\right)$. The symmetry $\eta$ of $R$ maps $C_{1}$ and $C_{2}$ each onto itself, for if $\eta$ interchanges $C_{1}$ and $C_{2}$, then every station of $R$ must be even, violating Lemma 2 . Since $R$ is $R$-connected, it follows that the sub-RR- 
systems $R_{1}$ and $R_{2}$ are both $R$-connected, and by Lemmas 2 and $3, R_{1}$ and $R_{2}$ each have two odd stations. Clearly $\eta$ induces symmetries on $R_{1}$ and $R_{2}$. There are two tracks of $C_{1}$ and two tracks of $C_{2}$ that are mapped onto themselves by $\eta$. These four tracks are all in different stations of $R$, implying that $R$ has four odd stations.

Conversely, suppose that $R$ has two towns and two companies and that conditions (1), (2) or (3) hold. We will choose integer values for the exponents $m, n, p$, and $q$, so that the resulting presentation $\varphi$ obtained from $R$ will satisfy the conditions of Lemma 1 . Once this is done we will be finished, since $\varphi$ corresponds to a spine of a closed manifold $M \in \mathfrak{\pi}_{R}$, which implies that $\Re_{R}$ consists of closed manifolds.

Let $\Phi=\left\langle a, b \mid W_{1}, W_{2}\right\rangle$ be the abstract presentation corresponding to $R$. After specific values for the exponents are chosen, we denote the resulting presentation by $\varphi=\left\langle a, b \mid w_{1}, w_{2}\right\rangle$.

In case (1) we have $\bar{W}_{1}=a^{m}$ and $\bar{W}_{2}=b^{n}$. Any choice for $m$ and $n$ subject to $m \neq 0$ and $n \neq 0$ will insure that $\bar{w}_{1} \neq 1$ and $\bar{w}_{2}$ is not a consequence of $\bar{s}_{1}$.

In case (2) we have $\bar{W}_{1}=a^{m}$ and $\bar{W}_{2}=a^{\delta m} b^{\alpha n+\beta(n+q)+\gamma q}$ for some integers $\alpha, \beta, \gamma$, and $\delta$. Note that the $a$-town can have only one nonempty station in view of the abstract relator $W_{1}=a^{m}$ and the fact that $R$ is a reduced RR-system. If we choose $m \neq 0$, then $\bar{w}_{1} \neq 1$. We can then assure that $\bar{w}_{2}$ is not a consequence of $\bar{w}_{1}$ by choosing $n$ and $q$ so that

$$
\alpha n+\beta(n+q)+\gamma q=(\alpha+\beta) n+(\beta+\gamma) q
$$

is odd (and therefore not 0 ). This can be done since exactly two of the four integers $\alpha, \beta, \gamma$, and $\delta$ are odd.

Now, for the case (3) we write $\bar{W}_{1}=a^{k_{1}} b^{k_{1}^{\prime}}$ and $\bar{W}_{2}=a^{k_{2}} b^{k_{2}^{\prime}}$ where

$$
k_{i}=\alpha_{i} m+\beta_{i}(m+p)+\gamma_{i} p=\left(\alpha_{i}+\beta_{i}\right) m+\left(\beta_{i}+\gamma_{i}\right) p
$$

and

$$
k_{i}^{\prime}=\alpha_{i}^{\prime} n+\beta_{i}^{\prime}(n+q)+\gamma_{i}^{\prime} q=\left(\alpha_{i}^{\prime}+\beta_{i}^{\prime}\right) n+\left(\beta_{i}^{\prime}+\gamma_{i}^{\prime}\right) q
$$

for each $i=1,2$. By Lemma 3 it follows that two of the four odd stations are attributable to $C_{1}$ and the other two to $C_{2}$. Thus for each $i=1,2$, exactly two of the six integers $\alpha_{i}, \beta_{i}, \gamma_{i}, \alpha_{i}^{\prime}, \beta_{i}^{\prime}$, and $\gamma_{i}^{\prime}$ are odd. We consider three cases depending on where each company has its odd stations (each company may have its two odd stations in the same or different towns); (a) each company has one odd station in each of the two towns; (b) each company has both its odd stations in the same town; and (c) one company has both its odd stations in one town and the other has its odd stations in different towns.

Corresponding to these three cases we may relabel the towns and the stations in the towns and reorder the companies (and hence the relators) so 
that of the following is satisfied:

(a) $\alpha_{1}, \beta_{2}, \alpha_{1}^{\prime}, \beta_{2}^{\prime}$ are odd,

(b) $\alpha_{1}, \beta_{1}, \alpha_{2}^{\prime}, \beta_{2}^{\prime}$ are odd, or

(c) $\alpha_{1}, \beta_{1}, \gamma_{2}, \gamma_{2}^{\prime}$ are odd.

In all cases we let $m=1, p=3, n=2, q=1$. Then it will follow that $k_{1}$ and $k_{2}^{\prime}$ are odd while $k_{1}^{\prime}$ is even. We then conclude that $\bar{w}_{1} \neq 1$ and that $\bar{w}_{2}$ is not a consequence of $\bar{w}_{1}$. This completes the proof.

2. Concluding remarks. First we observe that in Theorem 2 the RR-systems covered by cases (1) and (2) are equivalent. In case (2) the $a$-town has only one nonempty station, and we use the RR-Elimination Theorem of [3] to eliminate every $a$-syllable appearing in $W_{2}$. This leaves us with an equivalent RR-system of the type covered in case (1). Further, it is an easy matter to identify each of these manifolds as being a connected sum of two lens spaces.

The question of identifying the manifolds covered by case (3) of Theorem 2 is not a trivial matter and will be discussed in our next paper. It will be shown that no counterexample to the Poincaré Conjecture exists which arises from a reduced two-town $R R$-system with eleven or fewer tracks.

The importance of Theorem 2 is that it provides a highly efficient algorithm for generating spines of all closed orientable 3-manifolds of genus 2 . A computer program has been written which generates an abstract presentation corresponding to each RR-system covered by case (3) of Theorem 2 having twenty or fewer tracks. There are 607 such RR-systems. Using RR-multiplication, these systems have been classified into 137 equivalence classes of RR-systems.

The proof of Theorem 1 establishes that every compact orientable 3-manifold with a spine corresponding to a 2-town RR-system has an involution that induces an involution on the spine. This result has been previously established in [5] using somewhat different techniques.

\section{REFERENCES}

1. R. S. Stevens, Classification of 3-manifolds with certain spines, Trans. Amer. Math. Soc. 205 (1975), 151-166. MR 50 \# 11245.

2. R. P. Osborne and R. S. Stevens, Group presentations corresponding to spines of 3-manifolds. I, Amer. J. Math. 96 (1974), 454-471. MR 50 \#8529.

3. _ Group presentations corresponding to spines of 3-manifolds. II, Trans. Amer. Math. Soc. 234 (1977), 213-243.

4. L. P. Neuwirth, An algorithm for the construction of 3-manifolds from 2-complexes, Proc. Cambridge Philos. Soc. 64 (1968), 603-613. MR 37 \#2231.

5. J. S. Birman and H. M. Hilden, Heegaard splittings of branched coverings of $S^{3}$, Trans. Amer. Math. Soc. 213 (1975), 315-352.

Department of Mathematics, Colorado State University, Fort Collins, Colorado 80521 (Current address of R. P. Osborne)

Current address (R. S. Stevens): Analytic Services, Falls Church, Virginia 22041 